\title{
A new norlignan glycoside from Cestrum diurnum $\mathrm{L}$.
}

\author{
Khaled M. Mohamed ${ }^{a}$, Mostafa A. Fouad ${ }^{b, *}$, Katsuyoshi Matsunami ${ }^{\mathrm{c}}$, Mohamed S. Kamel, \\ and Hideaki Otsuka ${ }^{c}$ \\ ${ }^{a}$ Department of Pharmacognosy, Faculty of Pharmacy, Assiut University, Assiut 71526, Egypt \\ ${ }^{b}$ Department of Pharmacognosy, Faculty of Pharmacy, Minia University, Minia 61519, Egypt \\ ${ }^{c}$ Department of Pharmacognosy, Graduate School of Biomedical Sciences, Hiroshima \\ University, 1-2-3 Kasumi, Minami-Ku, Hiroshima 734-8553, Japan \\ E-mail:느ouad2000@yahoo.com
}

\begin{abstract}
From the methanolic extract of Cestrum diurnum L. leaves, a new 9-norlignan glucoside (cestrumoside) (1) was isolated in addition to six known glycosides (2-7). The structures of the isolated compounds were determined on the bases of NMR spectral analysis $\left({ }^{1} \mathrm{H} \mathrm{NMR},{ }^{13} \mathrm{C}\right.$ NMR, HSQC, HMBC, COSY) in addition to MS and CD to infer the stereochemistry of the compounds.
\end{abstract}

Keywords: Cestrum diurnum L., 9-norlignan, cestrumoside

\section{Introduction}

The genus Cestrum (Solanaceae) has many species that are used in the Chinese traditional medicine for the treatment of burns and swellings ${ }^{1}$. Several compounds including steroidal saponins, flavonoids, vitamin $\mathrm{D}_{3}$ derivative and lignans have been isolated from the genus Cestrum $^{1-7}$. From the methanolic extract of Cestrum diurnum L., we have reported the isolation and structure elucidation of new furostanol steroidal saponins ${ }^{8}$. As a continuation of our investigation on this species, a new 9-norlignan glycoside named cestrumoside (1) was isolated together with six known glycosides, berchemol-4'-O- $\beta$-glucopyranoside (2), liriodendrin (3), dehydrodiconiferyl alcohol-4-O- $\beta$-glucopyranoside (4), (+) and (-)-lyoniresinol 3a-O- $\beta$ glucopyranoside (5 and 6) and the megastigmane derivative citroside B (7) (Fig 1,3). The structures of the isolated compounds were verified by using NMR and MS spectral analysis. 


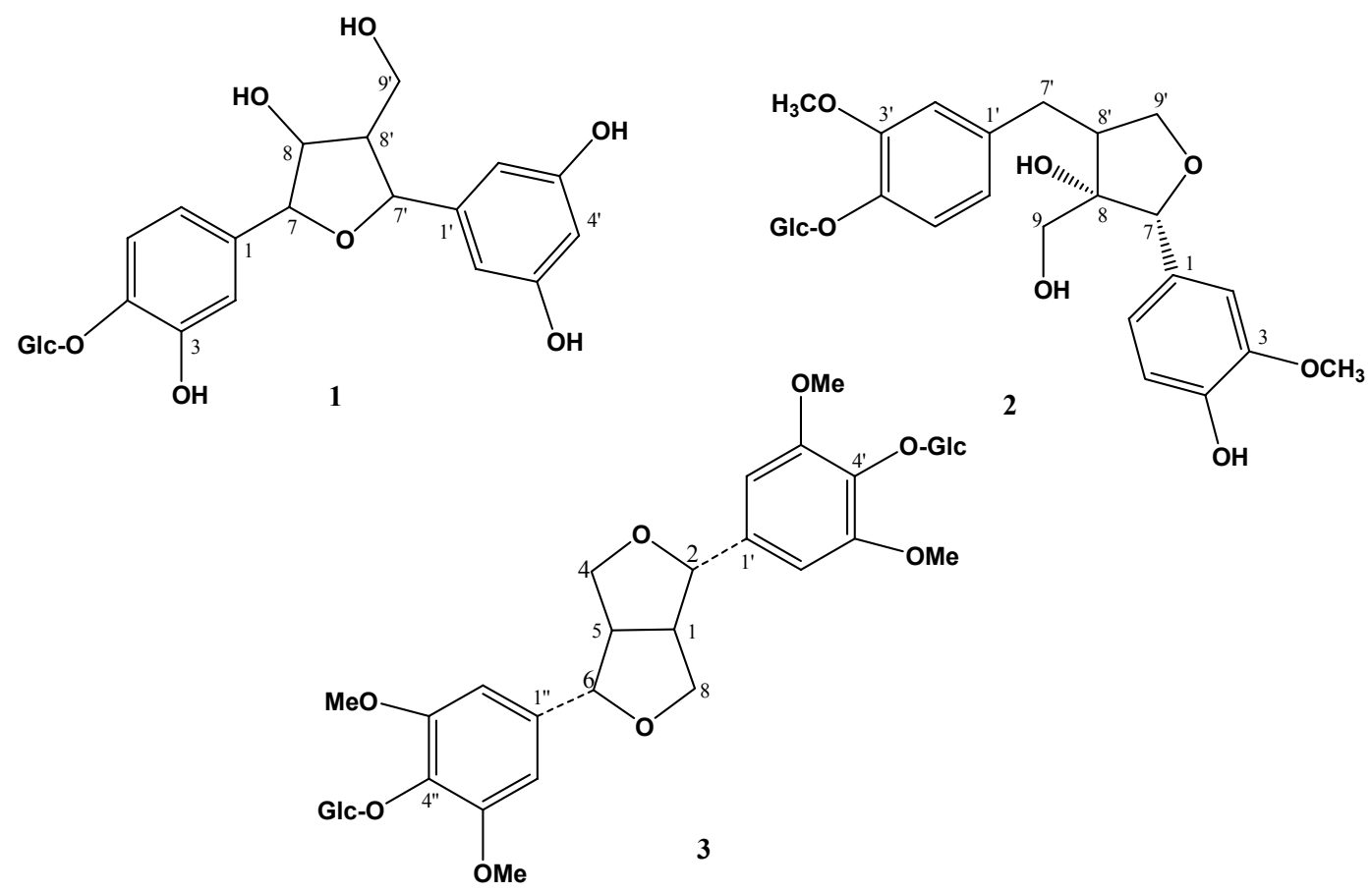

Figure 1. Structures of compounds 1, 2 and 3.

\section{Results and Discussion}

The aerial parts of Cestrum diurnum L. were collected from the plants cultivated in the Experimental Station of Faculty of Agriculture, Assiut University, Assiut, Egypt, in December 2004. The plant was kindly authenticated by Prof. Naeem El-Keltawy, Prof. of Horticulture, Faculty of Agriculture, Assiut University, Assiut, Egypt. A voucher sample was kept in the Herbarium of Pharmacognosy Department, Faculty of Pharmacy, Assiut University, Assiut, Egypt.

Compound 1 was isolated as a white amorphous powder. The HR-FABMS of 1 showed a molecular ion peak at $m / z 519.1472\left[\mathrm{M}+\mathrm{Na}\right.$ ] corresponding to a molecular formula $\mathrm{C}_{23} \mathrm{H}_{28} \mathrm{O}_{12} \mathrm{Na}$. The ${ }^{13} \mathrm{C}$, DEPT ${ }^{13} \mathrm{C}$ and ${ }^{1} \mathrm{H}$ NMR spectral data of $\mathbf{1}$ (Table 1) showed the presence of a signal at $\delta_{\mathrm{C}} 103.1$ with $\delta_{\mathrm{H}} 4.86(1 \mathrm{H}, d, J=7.1 \mathrm{~Hz})$ characteristic for the anomeric carbon and proton of a $\beta$-glucopyranosyl moiety. The preliminary inspections of the NMR spectral data of 1 showed the presence of three aromatic protons at $\delta_{\mathrm{H}} 6.96(1 \mathrm{H}, d, J=1.6 \mathrm{~Hz}, \mathrm{H}-2), 7.12(1 \mathrm{H}, d, J=8.3 \mathrm{~Hz}, \mathrm{H}-$ 5) and $6.92(1 \mathrm{H}, d d, J=8.3,1.6 \mathrm{~Hz}, \mathrm{H}-6)$ with $\delta_{\mathrm{C}} 112.7(\mathrm{C}-2), 117.8$ (C-5) and 120.9 (C-6), respectively as confirmed from HSQC spectrum indicating the presence of a 1, 3, 4-trisubstituted benzene ring (ABX system). 
Table 1. ${ }^{1} \mathrm{H},{ }^{13} \mathrm{C}$ NMR spectral data $\left(400,100 \mathrm{MHz}, \mathrm{CD}_{3} \mathrm{OD}\right)$ of compounds $\mathbf{1 , 2}$

\begin{tabular}{|c|c|c|c|c|}
\hline \multirow[t]{2}{*}{ Pos. } & \multicolumn{2}{|l|}{1} & \multicolumn{2}{|l|}{2} \\
\hline & $\delta_{\mathrm{H}}(\mathrm{JHz})$ & $\delta_{\mathrm{C}}$ & $\delta_{\mathrm{H}}(J \mathrm{~Hz})$ & $\delta_{\mathrm{C}}$ \\
\hline 1 & - & 137.6 & - & 133.4 \\
\hline 2 & $6.96,1 \mathrm{H}, d, J=1.6$ & 112.7 & $7.02,1 \mathrm{H}, d, J=1.8$ & 113.6 \\
\hline 3 & - & 150.6 & - & 147.6 \\
\hline 4 & - & 145.6 & - & 149.1 \\
\hline 5 & $7.12,1 \mathrm{H}, d, J=8.3$ & 117.8 & $6.71,1 \mathrm{H}, d, J=8.1$ & 116.3 \\
\hline 6 & $6.92,1 \mathrm{H}, d d, J=8.3,1.6$ & 120.9 & $6.85,1 \mathrm{H}, d d, J=8.1,1.8$ & 121.5 \\
\hline 7 & $4.25,1 \mathrm{H}, d, J=5.4$ & 87.2 & $4.88,1 \mathrm{H}, s$ & 85.5 \\
\hline 8 & $4.94,1 \mathrm{H}$, br.d,$J=5.0$ & 74.0 & - & 83.4 \\
\hline 9 & - & - & $3.59,3.80($ each $1 \mathrm{H}, d, J=11.5)$ & 64.6 \\
\hline $1^{\prime}$ & - & 132.9 & - & 134.7 \\
\hline $2^{\prime}$ & $6.71,2 \mathrm{H}, b r s$ & 104.1 & $6.80,1 \mathrm{H}, d, J=1.8$ & 113.7 \\
\hline $3^{\prime}$ & - & 154.7 & - & 150.5 \\
\hline $4^{\prime}$ & $6.87,1 \mathrm{H}, b r s$ & 116.7 & - & 146.0 \\
\hline $5^{\prime}$ & - & 154.7 & $7.12,1 \mathrm{H}, d, J=8.3$ & 117.7 \\
\hline $6^{\prime}$ & $6.71,2 \mathrm{H}$, brs & 104.1 & $6.64,1 \mathrm{H}, d d, J=8.3,1.8$ & 122.3 \\
\hline $7^{\prime}$ & $5.58,1 \mathrm{H}, d, J=5.9$ & 89.0 & $\begin{array}{l}3.08,1 \mathrm{H}, d d, J=12.7,3.2,{\mathrm{H}-7^{\prime}}_{\mathrm{a}} \\
2.49,1 \mathrm{H}, m, \mathrm{H}-7^{\prime}{ }_{\mathrm{b}}\end{array}$ & 35.0 \\
\hline $8^{\prime}$ & $3.77,1 \mathrm{H}, m$ & 55.5 & $2.56,1 \mathrm{H}, m$ & 52.0 \\
\hline $9^{\prime}$ & $\begin{array}{l}4.19,1 \mathrm{H}, m, \mathrm{H}-9^{\prime}{ }_{\mathrm{a}} \\
3.79,1 \mathrm{H}, m, \mathrm{H}-9^{\prime}{ }_{\mathrm{b}}\end{array}$ & 65.1 & $\begin{array}{l}3.72,1 \mathrm{H}, d d, J=7.5,4.9, \mathrm{H}-9^{\prime}{ }_{\mathrm{ax}} \\
4.06,1 \mathrm{H}, d d, J=7.5,6.4, \mathrm{H}-9^{\prime} \mathrm{eq}\end{array}$ & 72.2 \\
\hline $\mathrm{OMe}$ & - & - & $\begin{array}{l}3.83,3 \mathrm{H}, s \\
3.87,3 \mathrm{H}, s\end{array}$ & $\begin{array}{l}56.5 \\
56.8\end{array}$ \\
\hline Glc & & & & \\
\hline $1 "$ & $4.86,1 \mathrm{H}, d, J=7.1$ & 103.1 & $4.89,1 \mathrm{H}, d, J=7.4$ & 103.1 \\
\hline $2^{\prime \prime}$ & $3.48,1 \mathrm{H}, \mathrm{m}$ & 75.0 & $3.44,1 \mathrm{H}, \mathrm{m}$ & 75.0 \\
\hline $3 "$ & $3.85,1 \mathrm{H}, \mathrm{m}$ & 77.9 & $3.82,1 \mathrm{H}, \mathrm{m}$ & 77.9 \\
\hline $4 "$ & $3.70,1 \mathrm{H}, \mathrm{m}$ & 71.5 & $3.71,1 \mathrm{H}, \mathrm{m}$ & 71.5 \\
\hline $5 "$ & $3.44,1 \mathrm{H}, \mathrm{m}$ & 78.2 & $3.42,1 \mathrm{H}, \mathrm{m}$ & 78.2 \\
\hline 6" & $3.86,2 \mathrm{H}, \mathrm{m}$ & 62.6 & $3.80,2 \mathrm{H}, \mathrm{m}$ & 62.6 \\
\hline
\end{tabular}

Besides, the ${ }^{1} \mathrm{H},{ }^{13} \mathrm{C}$ NMR and HSQC showed a singlet signal at $\delta_{\mathrm{H}} 6.71\left(2 \mathrm{H}, b r \mathrm{~s}, \mathrm{H}-2^{\prime}, 6^{\prime}\right)$ with $\delta_{\mathrm{C}} 104.1\left(2 \mathrm{C}, \mathrm{C}-2^{\prime}, 6^{\prime}\right)$, a broad singlet signal at $\delta_{\mathrm{H}} 6.87\left(1 \mathrm{H}, \mathrm{br} s, \mathrm{H}-4^{\prime}\right)$ with $\delta_{\mathrm{C}} 116.9(\mathrm{C}-$ $\left.4^{\prime}\right)$ and two quaternary oxygenated aromatic carbons at $\delta_{\mathrm{C}} 154.7\left(2 \mathrm{C}, \mathrm{C}-3^{\prime}, 5^{\prime}\right)$ together with a quaternary aromatic carbons at $\delta_{\mathrm{C}} 132.9\left(\mathrm{C}-1^{\prime}\right)$ indicated the presence of a tri-substituted benzene ring. Furthermore, the ${ }^{1} \mathrm{H}$ and ${ }^{13} \mathrm{C}$ NMR spectra revealed the presence of three oxygenated methines at $\delta_{\mathrm{H}} 4.25(1 \mathrm{H}, d, J=5.4 \mathrm{~Hz}, \mathrm{H}-7)$ with $\delta_{\mathrm{C}} 87.2(\mathrm{C}-7), \delta_{\mathrm{H}} 5.58(1 \mathrm{H}, d, J=$ $\left.5.9 \mathrm{~Hz}, \mathrm{H}-7^{\prime}\right)$ with $\delta_{\mathrm{C}} 89.0\left(\mathrm{C}-7^{\prime}\right)$ and $\delta_{\mathrm{H}} 4.94(1 \mathrm{H}, b r . d, J=5.0 \mathrm{~Hz}, \mathrm{H}-8)$ with $\delta_{\mathrm{C}} 74.0(\mathrm{C}-8)$, in 
addition to a multiplet signal at $\delta_{\mathrm{H}} 3.77\left(1 \mathrm{H}, \mathrm{H}-8^{\prime}\right)$ with $\delta_{\mathrm{C}} 55.5\left(\mathrm{C}-8^{\prime}\right)$. The $\mathrm{H}-\mathrm{H}$ COSY analysis (Fig. 2a,b) showed spin system correlations between $\mathrm{H} 7-\mathrm{H} 8, \mathrm{H} 8-\mathrm{H} 8^{\prime}, \mathrm{H} 8^{\prime}-\mathrm{H} 7^{\prime}$ that indicated the presence of a tetrahydrofuran ring. The presence of a hydroxymethyl group at $\delta_{\mathrm{C}} 65.1\left(\mathrm{C}-9^{\prime}\right)$ with $\delta_{\mathrm{H}} 4.19\left(1 \mathrm{H}, m, \mathrm{H}-9^{\prime}{ }_{\mathrm{a}}\right)$ and $3.79\left(1 \mathrm{H}, m, \mathrm{H}-9^{\prime}{ }_{\mathrm{b}}\right)$ attached to $\mathrm{C}-8^{\prime}$ was proved from the $\mathrm{H}-\mathrm{H}$ COSY correlation (Fig. $2 \mathrm{a}, \mathrm{b}$ ) between $\mathrm{H} 8^{\prime}-\mathrm{H} 9^{\prime}{ }_{\mathrm{a}, \mathrm{b}}$.

The above-mentioned data suggested that compound $\mathbf{1}$ is a 9-norlignan glucoside ${ }^{9}$. The structural assignments of 1 was confirmed by HMBC analysis (Fig. 2a,b), in which an important correlation peaks were observed between both $\mathrm{H}-8$ and $\mathrm{H}-5$ with $\mathrm{C}-1$; H-8', H-9' and H-2',6' with C-7'. The attachment of the glucose moiety to $\mathrm{C}-4$ was confirmed by the presence of a correlation peak between the anomeric proton of the glucose with C-4 in the HMBC spectrum (Fig. 2a,b).

The position of the hydroxyl groups in compound $\mathbf{1}$ was confirmed from the HMBC analysis (Fig. 2a,b).

The relative trans configuration between the methine protons at C-7 and C-8; C-8 and C-8'; C-8' and C-7' were established by the 2D ROESY spectral analysis. Additionally, the configuration of $\mathrm{C}-7$ and $\mathrm{C}-7^{\prime}$ were characterized as $7 S$ and $7 ' S$, respectively by the $\mathrm{CD}$ spectrum $^{10-11}$.

On the basis of the above data, the structure of compound $\mathbf{1}$ including stereochemistry was concluded to be ( $\left.7 S, 8 R, 7^{\prime} S, 8^{\prime} R\right)$-9-norlignan-4-O-glucoside derivative.

To the best of our knowledge, this is the first report about the isolation of a 9-norlignan glycoside derivative from a natural source.

Compounds (2-7) (Fig. 1, 3) were identified by using different techniques of NMR spectral analysis and by comparison their NMR data and physical properties including optical rotation with those reported in literature as follows: the epoxy lignan glycoside berchemol-4'-O- $\beta$ glucopyranoside $(\mathbf{2})^{12,13}$, the lignan and neolignan glycosides liriodendrin $(\mathbf{3})^{14}$, dehydrodiconiferyl alcohol-4-O- $\beta$-glucopyranoside $\quad(\mathbf{4})^{15}, \quad(+)$-lyoniresinol 3a-O- $\beta$ glucopyranoside $(5)^{11}$ and (-)-lyoniresinol 3a-O- $\beta$-glucopyranoside $(\mathbf{6})^{11}$ and the megastigmane glucoside citroside $\mathrm{B}(7)^{16}$.

In conclusion, compound $\mathbf{1}$ is a new natural product, while known compounds (2-7) are reported here for the first time from the genus Cestrum.

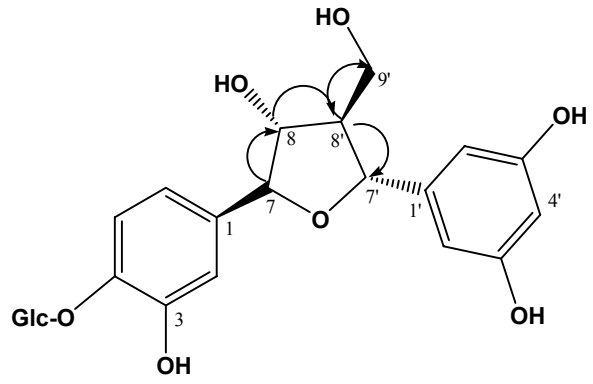

COSY correlations

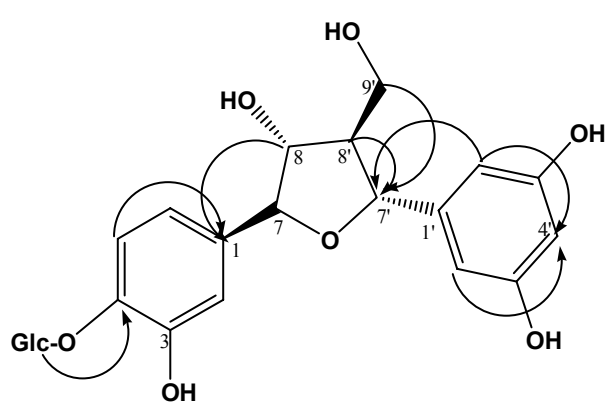

HMBC correlations

Figure 2a. H-H COSY and important HMBC correlations of compound $\mathbf{1}$. 


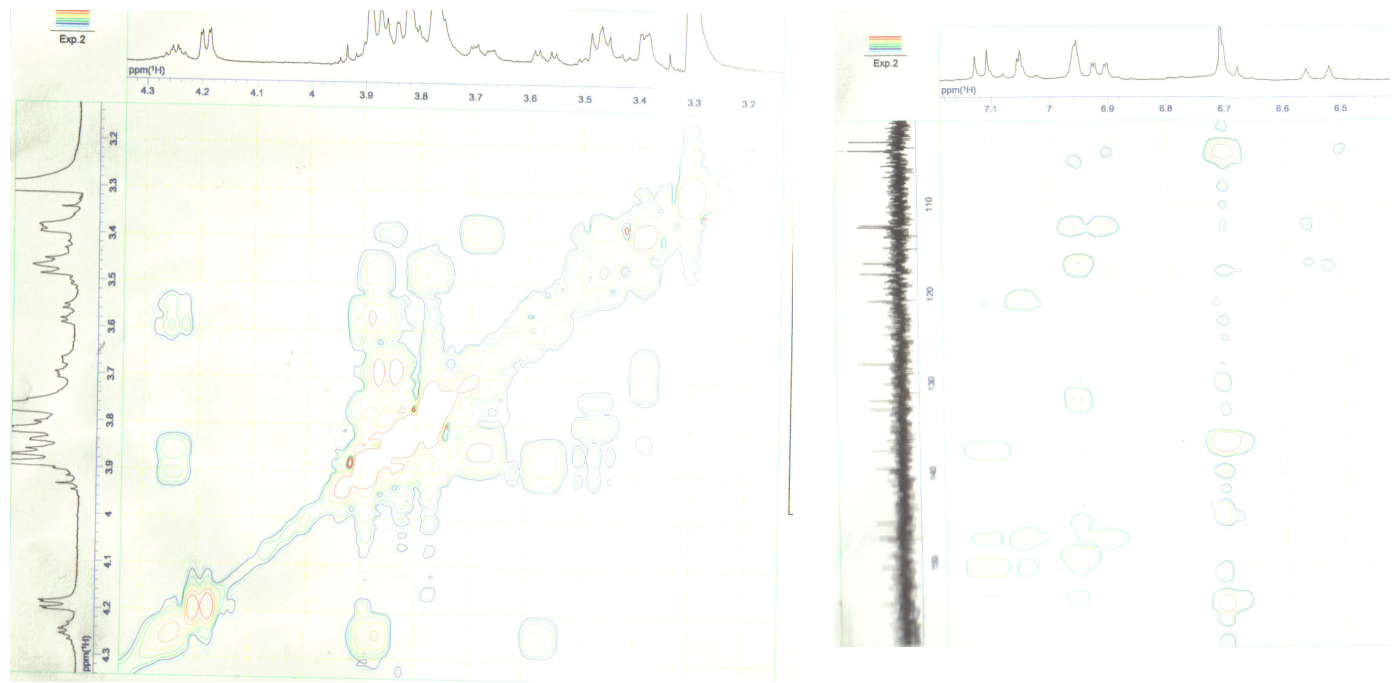

Figure 2b. Partial COSY and HMBC spectra of compound 1.

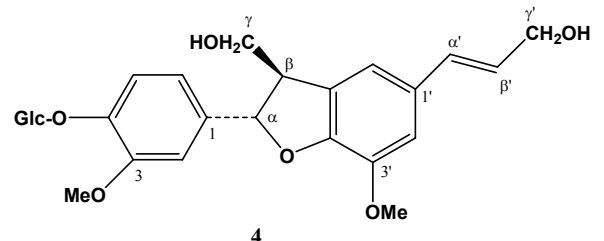

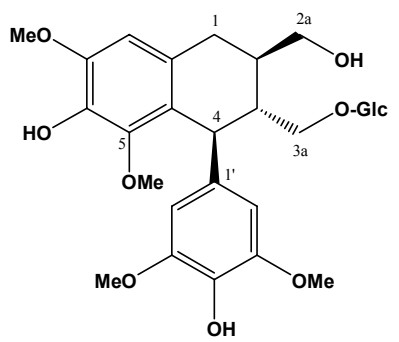

5

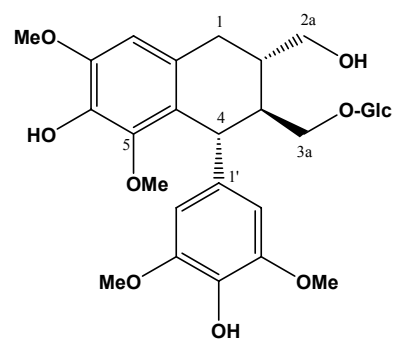<smiles>CC(=O)/C=C/C1[C@H](C)CC(O)C[C@]1(C)O</smiles>

Figure 3. Structures of compounds 4, 5, 6 and 7. 


\section{Experimental Section}

General Procedures. Optical rotations were measured on Union PM-101 automatic digital polarimeter. FT-IR spectra were recorded on a Horiba FT-710 spectrophotometer. HR-FABMS was recorded with JEOL JMS-SX 102 spectrophotometer. ${ }^{1} \mathrm{H}$ NMR and ${ }^{13} \mathrm{C}$ NMR spectra were measured on JEOL JNM A400 spectrophotometer (400 and $100 \mathrm{MHz}$, respectively) using TMS as an internal standard. Column chromatography was performed on Kieselgel 60 (60-230 mesh, Merck), Lichroprep RP-18 (Merck) and Diaion HP-20 (Mitsubishi). Preparative HPLC was carried out on columns of Polyamine and ODS (each 150x20 mm i.d., YMC) with JASCO PU1580 Pump and TOYO SODA RI-8000 refractive index detector. TLC was carried out with silica gel 60 pre-coated plates F-254s.

\section{Extraction and Isolation}

The air-dried powdered aerial parts of Cestrum diurnum (900 g) was exhaustively extracted with methanol at room temperature. The methanolic extract was concentrated under reduced pressure till dryness. The residue $(80 \mathrm{~g})$ was suspended in water and fractionated with $\mathrm{CHCl}_{3}$. The aqueous layer, after evaporation to a minimum volume, was subjected to a Diaion HP-20 CC and eluted with water, $\mathrm{MeOH}$ and finally with acetone. The residue of the $\mathrm{MeOH}$ eluate $(22 \mathrm{~g})$ was subjected to a silica gel $\mathrm{CC}$ using $\mathrm{CHCl}_{3}-\mathrm{MeOH}-\mathrm{H}_{2} \mathrm{O}$ (80:20: 2 to 65: 35: 5) gradient as eluting systems to give four fractions (Fractions I, II, III and IV). From fraction IV, the steroidal saponins cesdiurins I-IV were isolated using prep. HPLC with polyamine column and 75\%, 77\% and $80 \% \mathrm{MeCN}$ as a mobile phase where cesdiurin IV, III, I and II were obtained respectively as previously reported $^{8}$. On the other hand, Fraction II (1.3 g) was chromatographed on RP-18 CC using $30 \%$ to $70 \% \mathrm{MeOH}$ gradient as solvent systems, where 13 sub-fractions were obtained (F1 to F-13). F-2 (63 mg) was chromatographed on prep. HPLC using ODS column and 20\% $\mathrm{MeCN}$ as a mobile phase where compound 3 (38 mg) was obtained. F-5 (36 mg) was subjected to prep. HPLC using polyamine column and $80 \% \mathrm{MeCN}$ as a solvent system to afford compound 7 (8 mg). F-6 (115 mg) was chromatographed on prep. HPLC using Polyamine column and 82\% MeCN as a mobile phase where compounds $2(9 \mathrm{mg}), 5(18 \mathrm{mg})$ and $\mathbf{6}(15 \mathrm{mg})$ were isolated. F10 (48 mg) was chromatographed on prep. HPLC using Polyamine column and $85 \% \mathrm{MeCN}$ as a mobile phase where compound 4 (22 mg) was obtained. F-12 (33 mg) was subjected to prep. HPLC using ODS column and 25\% MeCN as a solvent system to afford compound 1 (17 mg).

Cestrumoside (1). A white amorphous powder, $[\alpha]_{\mathrm{D}}{ }^{23}-24.3(c 0.23, \mathrm{MeOH}) ; \mathrm{IR} v_{\max }($ film $) \mathrm{cm}^{-}$ ${ }^{1}$ : 3367, 2935, 1595, 1506, 1462, 1422, 1328, 1219, 1124, 832; UV $\lambda_{\max }(\log \varepsilon): 274.6$ (4.0), 226.8 (4.2); CD nm $(\Delta \varepsilon): 273$ (-3.42), 240 (+4.25), 220.8 (-4.66); HR-FABMS m/z: 519.1472 $\left(\mathrm{C}_{23} \mathrm{H}_{28} \mathrm{O}_{12} \mathrm{Na}\right.$ requires 519.1478) [M+Na].

Berchemol-4'-O- $\beta$-glucopyranoside (2). A white amorphous powder, $[\alpha]_{\mathrm{D}}{ }^{23}-36.5$ (c 0.19 , $\mathrm{MeOH}$ ); IR $\lambda_{\max }$ (film) $\mathrm{cm}^{-1}: 3367,2927,1600,1514,1425,1267,1222,1070,794$; UV $\lambda_{\max }$ (log $\varepsilon$ ): 278.8 (3.66), 229.2 (4.02); CD nm $(\Delta \varepsilon)$ : 276.5 (-0.69), 227.4 (-3.10), 215.5 (-3.57); HR-FABMS m/z: $561.1933\left(\mathrm{C}_{26} \mathrm{H}_{34} \mathrm{O}_{12} \mathrm{Na}\right.$ requires 561.1942) [M+Na]. 
Liriodendrin (3). A white amorphous powder, $[\alpha]_{\mathrm{D}}{ }^{23}-22.5$ (c 0.2 , pyridine); ${ }^{1} \mathrm{H}$ NMR

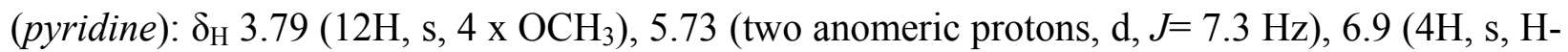
$\left.2^{\prime}, 6{ }^{\prime}, 2^{\prime}, 6^{\prime \prime}\right) .{ }^{13} \mathrm{C}$ NMR (pyridine): $\delta_{\mathrm{C}} 54.7(2 \mathrm{C}, \mathrm{C}-1,5), 56.7$ (4C, $\left.4 \mathrm{X} \mathrm{OCH}_{3}\right), 72.2(2 \mathrm{C}, \mathrm{C}-4$,

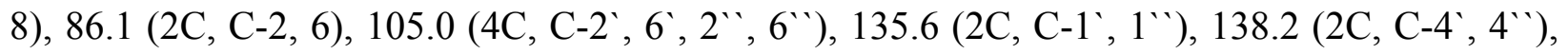
153.9 (4C, C-3’, 5’, 3’ , 5' '), 104.9 (2 anomeric carbons), 75.9, 78.3, 71.4, 78.6 and 62.6 (10C of the two glucose moieties).

Dehydrodiconiferyl alcohol-4-O- $\beta$-glucopyranoside (4). A white amorphous powder, $[\alpha]_{\mathrm{D}}{ }^{23}{ }_{-}$ 68.4 (c 0.5, MeOH); ${ }^{1} \mathrm{H}$ NMR (pyridine): $\delta_{\mathrm{H}} 3.83(1 \mathrm{H}, \mathrm{m}, \mathrm{H}-\beta), 3.83,3.60$ (each, 3H, s, $2 \mathrm{X}$ $\left.\mathrm{OCH}_{3}\right), 6.01(1 \mathrm{H}, \mathrm{d}, J=6.6 \mathrm{~Hz}, \mathrm{H}-\alpha), 6.54$, dt, $\left.J=5.4,15.9, \mathrm{H}-\beta^{`}\right), 6.86(1 \mathrm{H}, \mathrm{d}, J=15.9 \mathrm{~Hz}, \mathrm{H}-$ $\left.\alpha^{\prime}\right), 7.38(1 \mathrm{H}, \mathrm{d}, J=8.3 \mathrm{~Hz}, \mathrm{H}-5), 7.27(1 \mathrm{H}, \mathrm{d}, J=1.8 \mathrm{~Hz}, \mathrm{H}-2), 7.13(1 \mathrm{H}, \mathrm{dd}, J=1.8,8.3 \mathrm{~Hz}, \mathrm{H}-$ 6), 7.25, 7.09 (each, 1H, brs, H2 $\left.6^{\prime}\right), 5.59\left(1 \mathrm{H}, \mathrm{d}, J=6.8 \mathrm{~Hz}\right.$, anomeric proton). ${ }^{13} \mathrm{C}$ NMR (pyridine): $\delta_{\mathrm{C}} 136.6(\mathrm{C}-1), 111.2(\mathrm{C}-2), 150.2(\mathrm{C}-3), 147.6(\mathrm{C}-4), 116.4(\mathrm{C}-5), 118.9(\mathrm{C}-6), 88.1$

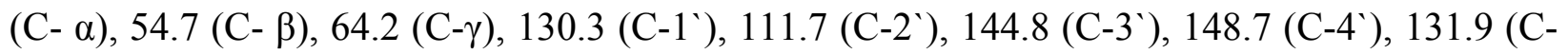
$\left.5^{`}\right), 116.4$ (C-6`), 129.0 (C- $\left.\alpha^{\prime}\right), 129.9\left(\mathrm{C}-\beta^{`}\right), 63.0\left(\mathrm{C}-\gamma^{\prime}\right), 55.9,56.2$ (2 X OCH $\left.\mathrm{OCH}_{3}\right), 102.2$ (anomeric carbon). $\mathrm{CD} \mathrm{nm}(\Delta \varepsilon): 284.5(-5.34701)$ as negative maximal.

(+)-Lyoniresinol 3a-O- $\beta$-glucopyranoside (5). A white amorphous powder, $[\alpha]_{\mathrm{D}}{ }^{23}+21.4(c$ 0.03, MeOH); ${ }^{1} \mathrm{H}$ NMR $\left(C D_{3} O D\right): \delta_{\mathrm{H}} 6.40(2 \mathrm{H}$, brs, H-2, 6$), 6.6(1 \mathrm{H}, \mathrm{s}, \mathrm{H}-8), 4.13(1 \mathrm{H}, \mathrm{d}, J=$ $7.84 \mathrm{~Hz}$, anomeric proton). ${ }^{13} \mathrm{C}$ NMR $\left(C D_{3} O D\right): \delta_{\mathrm{C}} 33.8(\mathrm{C}-1), 40.7(\mathrm{C}-2), 46.6(\mathrm{C}-3), 42.8(\mathrm{C}-$ 4), 147.5 (C-5), 139.3 (C-6), 148.7 (C-7), 107.9 (C-8), 130.2 (C-9), 126.3 (C-10), 139.4 (C-1'), 107.1 (C-2`), 149.0 (C-3`), 134.0 (C-4'), 149.0 (C-5'), 107.1 (C-6 '), 66.3 (C-2a), 72.1 (C-3a), 56.6, 56.9, 60.1 (4 $\left.\mathrm{X} \mathrm{OCH}_{3}\right), 104.3$ (anomeric carbon).

(-)-Lyoniresinol 3a-O- $\beta$-glucopyranoside (6). A white amorphous powder, $[\alpha]_{\mathrm{D}}{ }^{23}-71.4(c 0.03$, $\mathrm{MeOH}) ;{ }^{1} \mathrm{H}$ NMR $\left(C D_{3} \mathrm{OD}\right): \delta_{\mathrm{H}} 6.40\left(2 \mathrm{H}, \mathrm{brs}, \mathrm{H}-2{ }^{\prime}, 6\right), 6.6(1 \mathrm{H}, \mathrm{s}, \mathrm{H}-8), 4.13(1 \mathrm{H}, \mathrm{d}, J=7.84$ $\mathrm{Hz}$, anomeric proton). ${ }^{13} \mathrm{C} \mathrm{NMR}\left(\mathrm{CD}_{3} \mathrm{OD}\right): \delta_{\mathrm{C}} 33.8(\mathrm{C}-1), 41.3(\mathrm{C}-2), 46.7$ (C-3), $43.2(\mathrm{C}-4)$, 147.5 (C-5), 139.3 (C-6), 148.7 (C-7), 107.9 (C-8), 130.2 (C-9), 126.4 (C-10), 139.4 (C-1'), 107.2 (C-2`), 149.0 (C-3`), 134.0 (C-4'), 149.0 (C-5'), 107.2 (C-6 '), 66.3 (C-2a), 72.1 (C-3a), 56.6, 56.9, 60.1 (4 $\left.\mathrm{X} \mathrm{OCH}_{3}\right), 104.8$ (anomeric carbon).

Citroside B (7). A white amorphous powder, $[\alpha]_{\mathrm{D}}{ }^{23}-29.6(c 0.2, \mathrm{MeOH}) ;{ }^{13} \mathrm{C}$ NMR $\left(C D_{3} O D\right)$ : $\delta_{\mathrm{C}} 37.0$ (C-1), 49 (C-2), 63.8 (C-3), 48.1 (C-4), 78.7 (C-5), 119.1 (C-6), 200.7 (C-7), 101.4 (C-8), 212.9 (C-9), 26.6 (C-6), 26.7 (C-11), 30.0 (C-12), 32.5 (C-13), 98.7 (C-anomeric). ${ }^{1} \mathrm{H}$ NMR $\left(C D_{3} O D\right): \delta_{\mathrm{H}} 5.84(1 \mathrm{H}, \mathrm{s}, \mathrm{H}-8), 2.14(3 \mathrm{H}, \mathrm{s}, \mathrm{H}-10), 1.42$ (3H, s, H-13), 1.32 (3H, s, H-12), 1.11 (3H, s, H-11), 4.47 (1H, d, J=7.6, H-anomeric).

\section{Acknowledgements}

The authors are grateful to The Japan Society of The Promotion of Science (JSPS) for the financial support of this work and the Research Center of Molecular Medicine of the Hiroshima University School of Medicine, Japan, for NMR measurements. 


\section{References}

1. Mimaki, Y.; Watanabe, K.; Sakagami, H.; Sashida, Y.; J. Nat. Prod. 2002, 65, 1863.

2. Ahmed, V.U.; Baqai, F.T.; Fatima, I.; Ahmad, R. Phytochemistry 1991, 30, 3057.

3. Ahmed, V.U.; Baqai, F.T.; Ahmad, R. Phytochemistry 1993, 34, 511.

4. Ahmed, V.U.; Baqai, F.T.; Ahmad, R. Z. Naturforsch 1995, 50b, 1104.

5. Mimaki, Y.; Watanabe, K.; Ando, Y.; Sakuma, C.; Sashida, Y.; Furuya, S.; Sakagami, H. J. Nat. Prod. 2001, 64, 17.

6. Prema, T.P. and Raghuramulu, N. Phytochemistry 1994, 37, 677.

7. Abrosca B.D'; Dellagreca, M.; Fiorentino, A.; Golino, A.; Monaco, P.; Zarrelli, A. Natural Products Research 2006, 20, 293.

8. Fouad, M. A.; Mohamed, K. M.; Matsunami, K.; Kamel, M.S.; Otsuka, H.; Phytochemistry, in press.

9. Moss, G.P.; Pure and Applied Chemistry 2000, 72, 1493.

10. Sugiyama, M.; Nagayama, E.; Kikuchi, M. Phytochemistry 1993, 33, 1215.

11. Yusa, K.; Ide, T.; Otsuka, H.; Ogimi, C.; Hirata, E.; Takushi, A.; Takeda, Y. Phytochemistry 1997, 45, 611.

12. Schumacher, B.; Scholle, S.; Holzl, J.; Khudeir, N.; Hess, S.; Muller, C. J. Nat. Prod. 2002, 65, 1479.

13. Sakurai, N.; Nagashima, S.; Kawai, K.; Inoue, T.; Chem. Pharm. Bull 1989, 37, 3311.

14. Kobayashi, H.; Karasawa, H.; Miyase, T.; Fukushima, S. Chem. Pharm. Bull. 1985, 33, 1452.

15. Yoshizawa, F.; Deyama, T.; Takizawa, N.; Usmanghani, K.; Ahmad, M. Chem. Pharm. Bull. 1990, 38, 1927.

16. Umehara, K.; Hattori, I.; Miyase, T.; Ueno, A.; Hara, S.; Kageyama, C. Chem. Pharm. Bull. 1988, 5004. 